\title{
Uruguay: Análisis econométrico de la masa monetaria e inflación, 1985 - 2017
}

\section{Uruguay: Econometric analysis of the money supply and inflation, 1985 - 2017}

\author{
Lady Andrea León Serrano ${ }^{1}$ \\ http://orcid.org/0000-0001-5472-140X \\ Ilady@utmachala.edu.ec \\ Oliver Renato LLaguno Ayala ${ }^{2}$ \\ https://orcid.org/0000-0001-8517-7048 \\ Ollaguno1@utmachala.edu.ec \\ Bryan Davis Recalde Bravo 3 \\ https://orcid.org/0000-0002-3058-7629 \\ brecalde1@utmachala.edu.ec
}

Recibido: 07/12/2020, Aceptado: 26/03/2021

\section{RESUMEN}

El crecimiento caracterizado en los últimos años de Latinoamérica ha llevado analizar dos factores que afectan la sostenibilidad de las economías, como es la masa monetaria y la inflación, indicadores que generan volatilidad en los comportamientos por los niveles de ingresos de divisas y la repercusión en los precios de bienes y servicios. Uruguay es parte de las economías afectadas por las crisis económicas enfrentando períodos de recesión y recuperación. Por tal motivo, el objetivo principal consiste en determinar la incidencia de la inflación en la masa monetaria de Uruguay, periodo 1985-2017, la base de datos obtenida corresponde al Banco Mundial. La metodología es modelación econométrica y documental. Los resultados señalan que al incrementarse la masa monetaria aumenta la inflación o viceversa, demostrando que las variantes son estadísticamente significativas con un $R^{\wedge} 2$ de 0.862 . Las conclusiones establecen que, en los primeros años del periodo de estudio se observa un aumento agresivo de inflación por las circunstancias de capacidad del dinero en la economía uruguaya, sin embargo, el proceso disminuye en los siguientes años, las políticas económicas de largo plazo adoptadas han estado encaminadas en el fortalecimiento de la producción con controles en los precios y direccionamiento de fondos al sector empresarial.

Palabras clave: inflación, masa monetaria, regresión lineal simple, Uruguay

\author{
ABSTRACT \\ ${ }^{1}$ Universidad Técnica de Machala \\ ${ }^{2}$ Universidad Técnica de Machala \\ ${ }^{3}$ Universidad Técnica de Machala
}




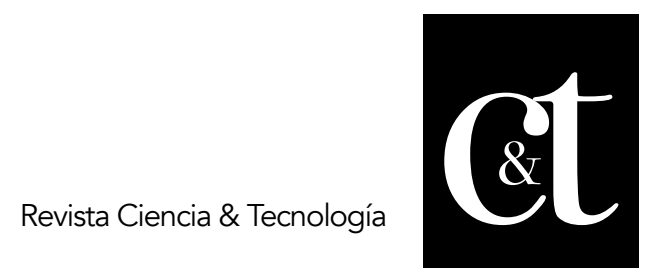

No. 30, 30 de abril de 2021

ISSN impreso: 1390 - 6321

ISSN online: 2661 - 6734

The growth characterized in recent years in Latin America has led to the analysis of two factors that affect the sustainability of the economies, such as the monetary mass and inflation, indicators that generate volatility in behavior due to the levels of foreign exchange earnings and the impact on prices of goods and services. Uruguay is part of the economies affected by the economic crises facing periods of recession and recovery. For this reason, the main objective is to determine the incidence of inflation in the money supply of Uruguay, period 1985-2017, the database obtained corresponds to the World Bank. The methodology is econometric and documentary modeling. The results indicate that as the money supply increases, inflation increases or vice versa, demonstrating that the variants are statistically significant with an $R \wedge 2$ of 0.862 . The conclusions establish that, in the first years of the study period, an aggressive increase in inflation is observed due to the circumstances of money capacity in the Uruguayan economy, however the process decreases in the following years, the long-term economic policies adopted have been aimed at strengthening production with price controls and directing funds to the business sector.

Keywords: inflation, money supply, simple linear regression, Uruguay

\section{Introducción}

El crecimiento económico es uno de los indicadores de mayor análisis por los países y cada día reflexionan sobre los hechos generados para delimitar acciones por los aumentos o disminuciones, los principales problemas macro se originan en los resultados de las políticas económicas, especialmente por los entes encargados en la determinación de instrumentos que mantengan el control global de la moneda para la sostenibilidad de las naciones.

América Latina ha experimentado cambios fluctuosos pro cíclicos y anti cíclicos de crisis financieras en los años treinta con la caída de la bolsa de valores de Estados Unidos, la política monetaria rezagada llevó al caos varias economías que dependían de ella, sin embargo, las intervenciones de conceptos keynesianos modelaron la nueva economía basada en la producción, tasa de interés y dinero. Los efectos comenzaron a relucir en Centro América y el cono Sur, en los precios exagerados de materia prima, la caída de exportaciones, aumento de importaciones, circunstancias que llevaron a Uruguay a elevar precios por los financiamientos internacionales, la oleada de divisas tuvo efectos directos en el consumo intermedio del sector productivo.

Por ende, es importante determinar la incidencia de la inflación en la masa monetaria en un período de largo plazo 1985 a 2017, la veracidad del trabajo de investigación se comprobó mediante un análisis econométrico corroborando que las variables son estadísticamente significativas, por lo tanto las dos variables tienen una relación directa, a medida que aumenta la inflación incrementa la oferta monetaria en bajos porcentajes o viceversa, esta realidad es el reflejo de las variaciones del mercado interno y externo.

León, Llaguno, Recalde. Uruguay: Análisis econométrico de la masa monetaria e inflación, 1985 - 2017 


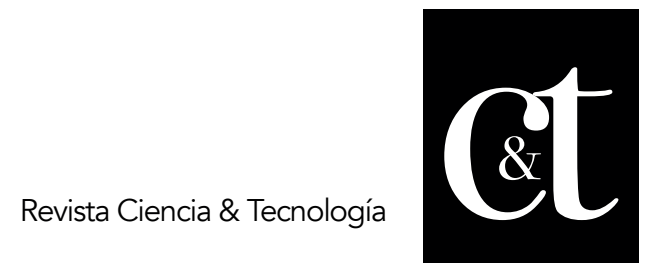

No. 30, 30 de abril de 2021

ISSN impreso: 1390 - 6321

ISSN online: 2661 - 6734

El tema se justifica por la investigación de dos variantes y las políticas económicas adoptadas en la mejora del comportamiento económico, los cambios de la inflación agresivos para terminar con porcentuales bajos en los últimos períodos representan tema de interés en el manejo monetario y fiscal. Las negociaciones monetarias representaban uno de los conjuntos básicos para apalancar las crisis internas, estas decisiones llevaron a generar recursos solventes en función de la demanda real de dinero. Es así, la estructura del artículo comprende diferentes secciones, comenzando con Introducción abarcando el aspecto global del entorno inflacionario y divisas, el segundo apartado con el Desarrollo desde los antecedentes junto a los hechos del tiempo de estudio, la tercera sección explica la Metodología de modelación econométrica, cuarto apartado los Resultados finales, quinta sección las Conclusiones generales y finalmente la bibliografía documenta los fundamentos teóricos.

\section{Metodología}

El análisis de la propuesta, parte desde un modelo econométrico, para Gujarati y Porter (2012) se encarga de introducir o incorporar aquella variable que se encuentra denominada como el error de perturbación (ui), estando en una población o residuos (ei) cuando se trabaja con muestras de la población, además podemos encontrar todos los elementos necesarios y acordes para ser estudiados desde el punto de vista empírico que difiere en un modelo económico. El tipo de modelo es Regresión Lineal Simple con Mínimo d Cuadrados Ordinarios (MCO), según (Astorga, 2014) explica la relación existente entre dos variables las cuales son consideradas como variable dependiente $(Y)$ y una sola variable independiente $(\mathrm{X})$.

La variable dependiente $(Y)$ es usada para describir o medir el problema estudiado, son aquellos datos o valores los cuales dependerán de los datos que tomen otra variable en pocas palabras serán explicadas por otro tipo de variable, se las conoce como: Variables dependientes, explicadas, predichas, respuesta, endógenas, resultado o variable controlada (Luceño y Gonzáles, 2015). La variable independiente $(X)$, desde el punto de vista cuantitativo son aquellos datos o valores que tratan de explicar, determinar o condicionar a otros datos de la variable dependiente $(Y)$, actúa como condicionante de otra variable y estos datos de $X$ explican a $Y$, se las conoce como: Variables independientes, predictora, regresora, exógena, covariante o variable de control (Cauas, 2015).

Mínimo Cuadrados Ordinarios también conocidos como mínimos cuadrados lineales permite encontrar los parámetros de una población dentro de un modelo de regresión lineal, se debe de considerar que los mínimos cuadrados ordinarios (MCO) cumplen con las características (MELI) es decir son Mejores Estimadores Lineales Insesgados por lo cual es el método más óptimo y apropiado para poder encontrar los Beta tanto $\left(B_{1}\right.$ y $\left.B_{2}\right)$ de una regresión lineal (Mballa y Sauceda, 2018). El Bo, conocido como beta sub cero, es aquel parámetro constante poblacional, se lo conoce como parámetro y en muestras toma el nombre de intercepto. $B_{1}$, conocido como beta sub uno $\left(B_{1}\right)$, es el parámetro de la población que indica la pendiente de la ecuación en la regresión, se considera que el $B_{1}$ siempre se encuentra acompañado de un coeficiente $X_{i}$, (Wooldridge, 2008). 


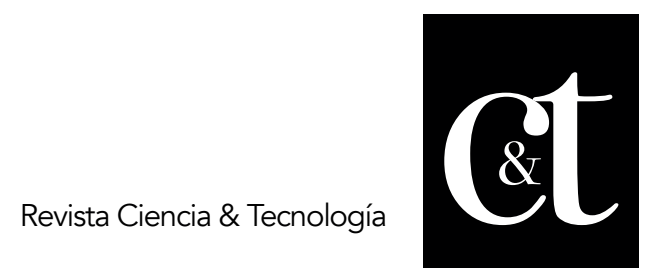

No. 30, 30 de abril de 2021

ISSN impreso: 1390 - 6321

ISSN online: 2661 - 6734

Para Laguna (2014), el Coeficiente de determinación $\left(R^{2}\right)$ es un indicador permite observar la importancia o explicación que puede dar la variable independiente $(X)$ en relación a la variable dependiente $(Y)$, es necesario mencionar que dicho coeficiente es la suma de los cuadrados de las variables Explicativas, dividido para la sumatoria de variables de cuadrados Totales. La significancia del $\left(R^{2}\right)$ oscila entre 0 y 1 , si tenemos un porcentaje entre $(0.70$ y 0.90) la significancia es muy buena caso contrario si el porcentaje es menor a (0.50) la significancia es muy baja llegando a la conclusión que la variable $X$ no explica nada a la variable (Y) (Stock y Watson, 2012), partiendo desde la siguiente fórmula:

Ecuación (2):

$$
R^{2}=\frac{\sum(\widehat{Y} x-\bar{Y})^{2}}{\sum(Y-\bar{Y}) x^{2}}
$$

Para el presente estudio, la variable inflación es (dependiente) y la masa monetaria es (independiente), mediante el coeficiente de determinación se explica si son significativas para el modelo, considerando las bases teóricas se representa con la siguiente ecuación:

Ecuación (3):

$y=\beta 0+\beta 1 X 1+u i$

Finalmente, los resultados plantean la aceptación del modelo mediante la corroboración con prueba de normalidad.

\section{Resultados y discusión}

La interpretación histórica de la magnitud y persistencia de los déficits fiscales en el tiempo son tópicos de estudio en el ámbito teórico y empírico, por los efectos de la deuda externa, a pesar de las expansiones monetarias la consistente inflación ha generado en diferentes períodos de Latinoamérica inestabilidades macroeconómicas (Barquero y Loaiza, 2017). Para (Fisher y Easterly, 1990) la rápida inflación es casi siempre un fenómeno fiscal y el control de la inflación ha requerido de coordinación entre política monetaria y fiscal.

La producción industrial mundial de los bienes de capital se mantuvo pasiva por la contracción de la actividad manufacturera de china y la inflación global afectada por la crisis se mantuvo en un promedio bajo de 0,3\% como consecuencia de la caída de los precios de las materias primas. Para los casos de Brasil, Colombia y Rusia, las depreciaciones de la moneda neutralizaron gran parte los precios internacionales (León, 2018).

En Latinoamérica, específicamente en Uruguay, una economía pequeña, que ocupó lugares destacados en el ranking mundial de ingreso per cápita desde los años 1930 en adelante, no ha dejado de divergir respecto a los países industrializados. En este devenir histórico ha alternado períodos de crecimiento con otros de fuerte retracción, dando por resultado una economía volátil en sus diversos frentes -PIB, inversión, exportaciones, términos de intercambio- y de bajo crecimiento.

4

León, Llaguno, Recalde. Uruguay: Análisis econométrico de la masa monetaria e inflación, 1985 - 2017 


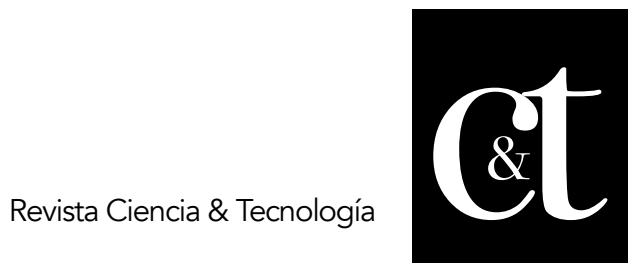

No. 30,30 de abril de 2021

ISSN impreso: 1390 - 6321

ISSN online: 2661 - 6734

Esa débil performance se expresó en el propio sistema de precios y durante la segunda mitad del siglo XX, Uruguay se transformó en una economía de alta y muy alta inflación (Brum, Román, y Willebald, 2013). Dos procesos fundamentales surgieron durante 1940 y 1960, empezando el primero con un significativo control de la oferta monetaria en las decisiones de política cambiaria, y el segundo destacado por los cambios introducidos en el sistema de emisión hacia un régimen de mayor flexibilidad. El Banco de la República de Uruguay adoptó la conducción de la política monetaria, debido que antes de 1960, la autoridad monetaria estaba repartida entre el Poder Legislativo y el Poder Ejecutivo, luego de la llegada de la crisis de Estados Unidos, el aparato financiero internacional comenzó atenuar el panorama nacional instaurándose un sistema de control a través de la creación del Contralor de Cambios, abandonando definitivamente el patrón oro. Los efectos de la crisis se reflejaron sobre todo en tres resultados de la economía: desequilibrio externo por el déficit de la balanza de pagos; caída del nivel de actividad; desajustes internos como la depreciación de la moneda y el déficit fiscal (Universidad de la República, 2010).

A pesar que se estimó una demanda de dinero con una elasticidad de ingreso unitaria, es posible identificar varios cambios de portafolio de los agentes económicos que impactaron significativamente en el grado de monetización de la economía, esto coincidió con un aumento importante de la inflación para cubrirse de la pérdida de valor de la moneda. Esta etapa transcurrida desde la década de 1960 hasta 1980 contrajo un nuevo período de remonetización (Brum, Román, y Willebald, 2013).

El modelo de desarrollo se sujetó a cambios, tal es así, en 1981 hasta 1989 se planteó como una inserción internacional vigilada por instituciones externas, la crisis de la deuda llevó a salidas promovidas por el Fondo Monetario Internacional a través del crédito generando aumento de la oferta monetaria para Uruguay, caracterizado por dos dimensiones básicas: papel del Estado y financiamiento del desarrollo. Dicha propuesta se mantuvo apegado a las importaciones basadas en los commodities derivados de recursos naturales de bajo valor agregado, es decir se caracterizaron por el redescubrimiento de la región, comenzando aumentar los altos niveles de inflación en un período de crisis promovido por los cambios macroeconómicos (Mañan, 2010). Terminando 1990 se implementa un nuevo programa de estabilización de precios, de carácter gradualista, luego, de anuncios a periódicos de las autoridades y el establecimiento de una banda de flotación cambiaria.

Dicho esquema siguió un derrotero similar al de sus antecesores y con la devaluación de junio de 2001 se habría llegado a un nuevo fracaso en la historia de los planes de estabilización de Uruguay. Sin embargo, la fuerte devaluación no significó, como en el pasado, una nueva y descontrolada escalada inflacionaria. Desde entonces, los hacedores de la política monetaria no volvieron a hablar del programa de estabilización y, en su lugar, se remitieron a los objetivos de todo banco central en preservar el valor de la moneda, mantener la estabilidad de precios, y crear las condiciones necesarias para la estabilidad del sistema financiero. Para ello, ha habido un traslado en el uso del ancla nominal pasando, primero, del tipo cambio hacia agregados monetarios $y$, luego, a la propia tasa de inflación con la tasa de interés como instrumento de política (Brum, Román, y Willebald, 2013). 


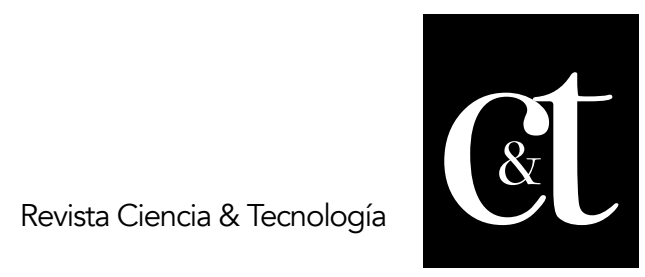

No. 30, 30 de abril de 2021

ISSN impreso: 1390 - 6321

ISSN online: 2661 - 6734

Debe tenerse en cuenta, que durante 2002-2008 en Uruguay la principal vinculación de sus componentes con los diferentes agentes de la economía fue: el circulante más dependiente del comportamiento de las familias; los depósitos a la vista más dependientes del comportamiento de las empresas, lo que a su vez fue asociado a los ciclos de la actividad productiva (Parejo, 2015).

En este caso la distorsión introducida por el período de crisis bancaria se remitió a una menor caída de la demanda de circulante de depósitos a la vista, dado la falta de confianza en el sistema financiero, y presumiblemente, por la mayor caída inicial en el nivel de actividad que en el ingreso nacional bruto disponible. No se descarta que haya incidido en este comportamiento una reestructuración del dinero mantenido por las empresas hacia un mayor peso del circulante, no disponiéndose de información al respecto. La mayor preferencia por el circulante también puede vincularse al crecimiento de la actividad informal típicamente asociada a los períodos de crisis. Además, en los primeros meses que sucedieron al feriado bancario se elevó la preferencia del circulante por motivos precautorios (Gómez, 2017).

Para 2009, la inflación anualizada cerró en 7\%, cumplió el rango meta fijado por el Gobierno debido a la política monetaria contractiva ejecutada, esto significó un decrecimiento de la inflación y el aumento de los salarios mínimos del empleo formal en los últimos años, a pesar de la no mejora de los ingresos de los trabajadores no formales fue afectado contemporáneamente por las variables exógenas del sistema. La heterogeneidad del crecimiento estuvo acompañada de la inseguridad macroeconómica de tasas de inflación elevadas e inestables por las distorsiones en los retornos de inversión, incremento la incertidumbre respecto a retornos futuros y reducción de costos de inversión. En 2010 hasta mediados del 2014 demostraron un decrecimiento de la masa monetaria sobre el crecimiento a largo plazo de 22 a 19 puntos y un movimiento constante en la inflación en 8\% (Banco Interamericano de Desarrollo (BID), 2015).

En la siguiente gráfica observamos, las variaciones de la inflación en un rango histórico de corte 1870-2010, la tendencia verifica la inestabilidad sometida a partir de 1955. Es decir, a mayor varianza en la rentabilidad del dinero mayor volatilidad de la inflación, los picos significativos especialmente de 1985 deducen a la profundización de la desmonetización en Uruguay como se observa en la figura 1. Vinculada a la desviación estándar del componente cíclico de la inflación (1870-2010).

León, Llaguno, Recalde. Uruguay: Análisis econométrico de la masa monetaria e inflación, $1985-2017$ 


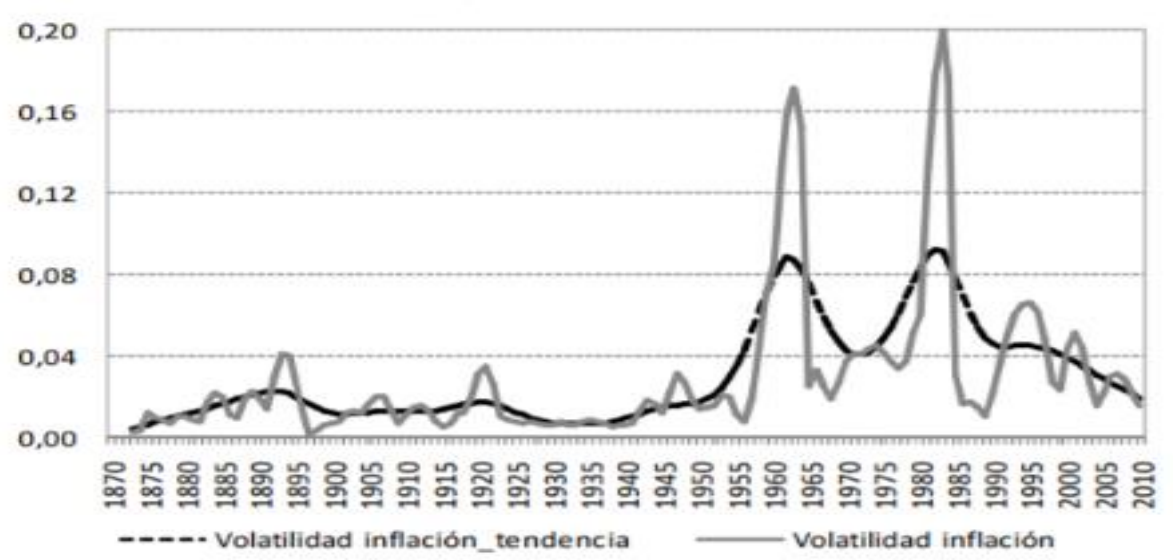

Fig. 1. Volatilidad de la inflación. Brum, Román y Willebald (2013).

Fuente: elaboración propia

Uruguay llegó a un crecimiento más fuerte del PIB que en años previos como en el 2015, aunque el aumento del desempleo se detuvo, la aplicación de políticas monetarias restrictivas y la apreciación del tipo de cambio fueron instrumentos vitales para el descenso de la inflación como lo fijado por el Banco Central en 2016 y 2017. Con este paso, las condiciones macroeconómicas mejoraron de manera estable y sostenida para la deuda pública, las tasas de interés bajaron en $2 \%$ impulsado por el consumo privado por la consecuente estabilidad de los precios, es así, los acuerdos salariales nominales en este periodo siguieron en aumento. El ajuste periódico del rango de preferencia para el crecimiento de la masa monetaria permitió adecuarse a la demanda de dinero y conciliar la consolidación fiscal y monetaria basada en la inversión (Fondo Monetario Internacional (FMI), 2017).

Las políticas del Gobierno de Uruguay se enfocaron en mejorar los niveles de inflación a un rango meta de 3 a $8 \%$. Para eso, la remonetización de la economía y el aumento de la demanda de dinero en paridad con la oferta monetaria. En la Tabla 01 se observa los datos de las variables de investigación, al principio en 1985 inicia con una fuerte inyección de dinero en la economía por los créditos externos versus el efecto en el aumento del nivel de precios, este comportamiento toma variaciones inestables desde 1991 hasta 2007, terminando en 6 puntos la inflación ante el $1 \%$ de oferta monetaria de disminución, la relación es directa según realidad de la economía Uruguaya, están tendencias a la baja o aumento en bajos porcentajes, han demostrado la fortaleza institucional en la toma de decisiones, que maneja como base de Datos: Inflación y Masa Monetaria. 


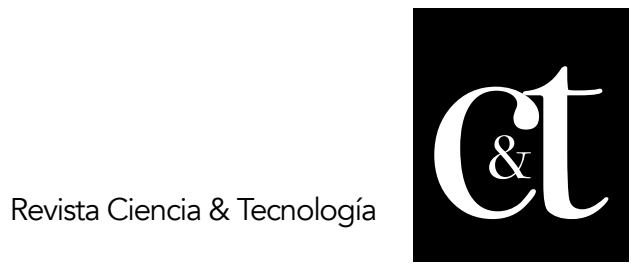

No. 30, 30 de abril de 2021

ISSN impreso: 1390 - 6321

ISSN online: 2661 - 6734

Tabla1. Uruguay 1985-2017. Banco Mundial (2019)

\begin{tabular}{|c|c|c|c|c|c|}
\hline Años & $\begin{array}{c}\text { Masa } \\
\text { Monetaria } \\
\text { (\%) } \\
\text { Anual) }\end{array}$ & $\begin{array}{c}\text { Inflación } \\
\text { \% Anual) }\end{array}$ & Años & $\begin{array}{c}\text { Masa } \\
\text { Monetaria } \\
\text { \% Anual) }\end{array}$ & $\begin{array}{c}\text { Inflación } \\
(\% \\
\text { Anual) }\end{array}$ \\
\hline 1985 & 57,92 & 72,22 & 2002 & 25,92 & 13,97 \\
\hline 1986 & 93,52 & 76,38 & 2003 & 15,19 & 19,37 \\
\hline 1987 & 56,53 & 63,56 & 2004 & $-3,04$ & 9,15 \\
\hline 1988 & 82,02 & 62,19 & 2005 & 0,02 & 4,69 \\
\hline 1989 & 103,51 & 80,44 & 2006 & 11,6 & 6,39 \\
\hline 1990 & 116,36 & 112,52 & 2007 & 3,8 & 8,11 \\
\hline 1991 & 81,85 & 101,97 & 2008 & 28,56 & 7,87 \\
\hline 1992 & 50,50 & 68,45 & 2009 & $-2,64$ & 7,06 \\
\hline 1993 & 36,51 & 54,10 & 2010 & 22,14 & 6,69 \\
\hline 1994 & 42,18 & 44,73 & 2011 & 17,99 & 8,09 \\
\hline 1995 & 36,87 & 42,24 & 2012 & 9,95 & 8,09 \\
\hline 1996 & 34,10 & 28,34 & 2013 & 19,18 & 8,57 \\
\hline 1997 & 28,64 & 19,81 & 2014 & 19,33 & 8,87 \\
\hline 1998 & 26,64 & 10,81 & 2015 & 23,81 & 8,66 \\
\hline 1999 & 12,97 & 5,65 & 2016 & 3,94 & 9,63 \\
\hline 2000 & 9,54 & 4,76 & 2017 & 1,91 & 6,21 \\
\hline 2001 & 14,57 & 4,35 & & & \\
\cline { 1 - 2 } & & & & & \\
\hline
\end{tabular}

Fuente: elaboración propia

Las explicaciones teóricas de la estabilidad de los precios siempre han sido prioridad de la política monetaria referente al crecimiento sustentable y mantenimiento del poder de compra de la moneda nacional, a pesar de la controversia sobre el origen de la inflación, la literatura maneja un amplio conjunto de condiciones que puede incidir en el nivel de precios. Simplificando, las escuelas de pensamiento actualmente dominante en la interpretación del fenómeno inflacionario pueden identificarse como monetarista y neokeynesiana. Desde la visión monetarista, la inflación es explicada fundamentalmente por la oferta monetaria por encima de la demanda real de dinero por cambios en sus fundamentos; desde las expectativas neokeynesianas identifican diferentes tipos de demanda, esto ocurre cuando el exceso de demanda agregada supera la oferta disponible, generando un aumento de los gastos del gobierno o sector privado (Brum, Román, y Willebald, 2013).

La inflación y la oferta monetaria se relacionan relativamente con la teoría cuantitativa del dinero, por el aporte de algunos economistas clásicos y neoclásicos, como: John Maynard Keynes, David Ricardo, David Hume, Irving Fisher y Milton Friedman (Chraki, 2018).

8

León, Llaguno, Recalde. Uruguay: Análisis econométrico de la masa monetaria e inflación, 1985 - 2017 


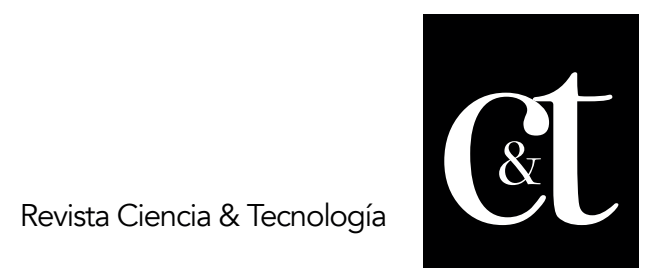

No. 30, 30 de abril de 2021

ISSN impreso: 1390 - 6321

ISSN online: 2661 - 6734

Esta teoría explica la relación de las variables con el nivel de producción (Mankiw, 2014). Usa como supuesto la velocidad de dinero (V) como un valor fijo u exógeno y la renta o producción $(Y)$ predeterminado de manera exógena por otras variables (Lage, 2014). Al existir una relación directa entre la oferta monetaria (M) y precios ( $P$ ), se produce un aumento de la oferta u masa monetaria (M) y para que se pueda mantener la igualdad de la ecuación deberá obligatoriamente aumentar el nivel de precios $(P)$ dado que la velocidad del dinero y la producción son fijos y viceversa.

Los cuestionamientos de Ludwig Von Mises afirmaba que dicha teoría tenía en cierto sentido algo de verdad pero que la inflación no puede ser explicada por un modelo simple, pues existen más variables que explican el fenómeno de la inflación (Ravier, 2017).

Teóricamente, la Masa Monetaria, es un indicador macroeconómico también conocido como oferta monetaria, es el dinero total o disponible que se encuentra en circulación en un tiempo determinado dentro de una economía, considera las monedas, billetes y cheques. Se distingue por las siguientes variables $M_{0}, M_{1}, M_{2}$, $\mathrm{M}_{3}$ y $\mathrm{M}_{4}$ (Romo y Campi, 2018). $\mathrm{M}_{0}$ : Es el dinero o moneda física en manos del público, más todas las cuentas bancarias que se depositan en los Bancos centrales, correspondiente a billetes y monedas emitidos por los bancos centrales; $M_{1}$ : Son los depósitos de dinero en las cuentas corrientes por parte de los ciudadanos y empresas, este tipo de dinero puede ser transferible por medio de cheques, es decir es el efectivo en manos del público más depósitos a la vista; $M_{2}$ : Llamado liquidez total, es $M_{1}$ más cuentas de ahorro teniendo en consideración que son depósitos existentes en el corto plazo, se identifica así: $M_{2}=M_{1}+$ Depósitos disponibles con preaviso hasta tres meses + Depósitos a plazo hasta dos años; $M_{3}$ : Es un agregado determinado por $M_{2}$ más todos los tipos de depósitos existentes y a su vez se incluye todos los depósitos bancarios en el largo plazo, se lo conoce como $\mathrm{M}_{2}+$ todos los demás tipos de certificados de depósito, depósitos en moneda extranjera; $\mathbf{M}_{4}$ :

También conocido como un agregado, aquí se considera la suma de $M_{3}$ y todas las emisiones de bonos, letras y pagarés conocidos como (cuasi dinero), determinado por $M_{3}+$ Bonos, Letras y Pagarés (Mcleay, Radia, y Thomas, 2015). Es así, $M_{1}, M_{2}, M_{3}$ y $M_{4}$ son conceptos que suman al dinero bancario de tal manera que la oferta monetaria está regida por el sistema bancario y por las decisiones que toman los bancos centrales (Campoverde, 2019). Otra de las variables de estudio es la Inflación, según (Calderón, 2015), es un agregado que explica el alza generalizado de los precios de los bienes y servicios representativos de un país conocido como canasta familiar. Para (Vallejo, 2016) considera el mejor escenario para una economía una inflación baja y controlada mediante tasas de interés y políticas monetarias.

La ecuación para el cálculo de la inflación, se menciona a continuación:

Ecuación (1):

$$
\text { Tasa de Inflacion }=\frac{I P C \text { actual }- \text { IPC base }}{I P C \text { actual }} * 100
$$




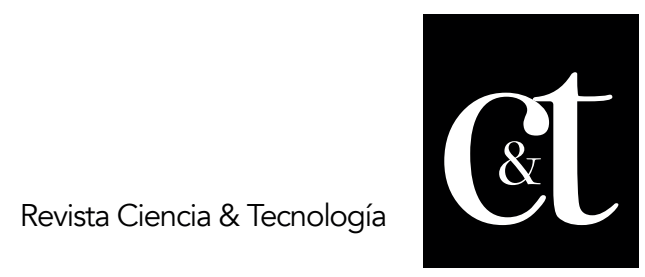

No. 30, 30 de abril de 2021

ISSN impreso: 1390 - 6321

ISSN online: 2661 - 6734

Al aumentar el Índice de Precios al Consumidor (IPC) existe inflación y en la medida que disminuye se produce deflación. Luego del recorrido histórico de la economía uruguaya en el tiempo de estudio y comprendiendo las bases conceptuales de las variables, el presente estudio permitió comprender que el proceso inflacionario en el largo plazo es un fenómeno esencialmente monetario por efectos de la oferta monetaria.

Un estado sin inflación, tampoco es un bien para la economía, la inflación es un indicador que controla los niveles de precios, fija salarios y permite mecanismos monetarios que actúen para manejar el exceso de liquidez, en el caso de Uruguay actualmente está fortalecida por los indicadores macroeconómicos, que han mejorado la tendencia histórica de inflaciones galopantes a ser uno de los países latinoamericanos con porcentajes de inflación controlables en base a la masa monetaria moderada sin interrumpir la inversión nacional y extranjera. El objetivo de la investigación consiste en determinar la incidencia de la inflación en la masa monetaria de Uruguay, periodo 1985-2017, los datos fueron obtenidos de la base de datos del Banco Mundial.

Tabla 2. Inflación y Masa Monetaria

Matriz General Modelo de Regresión Lineal Simple con Mínimos Cuadrados Ordinarios: Uruguay 1985-2017

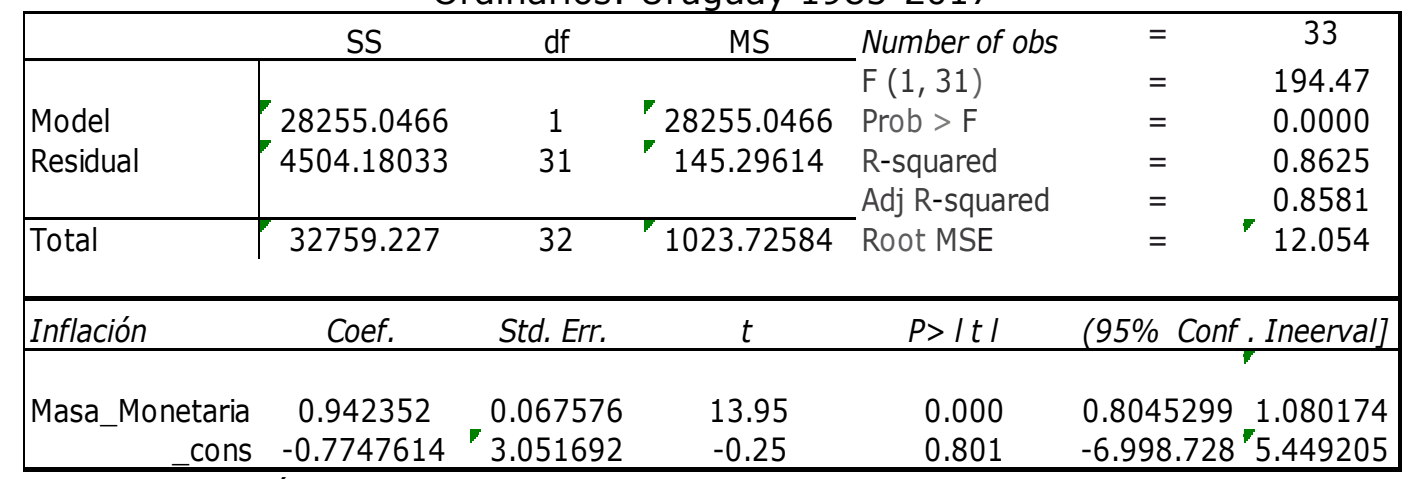

Fuente: elaboración propia

En la Tabla 02. se expone los resultados del análisis de regresión lineal simple con $\mathrm{MCO}$, con el factor masa monetaria, obteniendo una probabilidad $=0.000$ afirmando que un valor por debajo del nivel del error admisible $5 \%$ considera que la variable independiente es estadísticamente significativa para explicar la dependiente.

El valor del coeficiente de determinación ajustado es igual 0.8581 define que la variable explicativa $X$ escogida es factible y se relaciona con la variable explicada Y. De acuerdo a lo anterior, se deriva la siguiente ecuación:

Ecuación (4):

Inflación $=-0.7747614+0.942352$ Masa Monetaria + ui

León, Llaguno, Recalde. Uruguay: Análisis econométrico de la masa monetaria e inflación, $1985-2017$ 


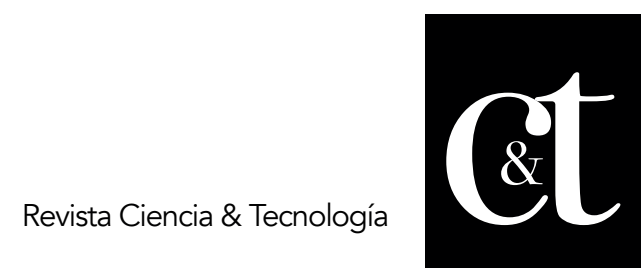

No. 30, 30 de abril de 2021

ISSN impreso: 1390 - 6321

ISSN online: 2661 - 6734

La interpretación, demuestra que por cada $1 \%$ de incremento en la Masa Monetaria, aumentará la Inflación en $0.9423 \%$ o viceversa.

Tabla 3. Test de comprobación

Matriz de Supuestos Modelo de Regresión Lineal Simple: Uruguay 1985-2017

\begin{tabular}{|c|c|}
\hline Heterocedasticidad & Normalidad \\
\hline Breusch-Pagan $=0.0848$ & Jarque-Bera $=0.4056$ \\
\hline
\end{tabular}

Fuente: elaboración propia

El supuesto de heterocedasticidad mediante la prueba Breusch-Pagan alcanza un resultado de 0.0848 , siendo menor que el valor crítico dado en la tabla de $x 2$, determinando que los datos residuales están distribuidos homocedásticamente resultando significativo para el modelo.

El segundo supuesto es la prueba Jarque Bera con un valor $=0.4056$, resultado mayor a 0.05, significa que los errores en el modelo siguen una distribución normal, revisando el respectivo diagrama de dispersión.

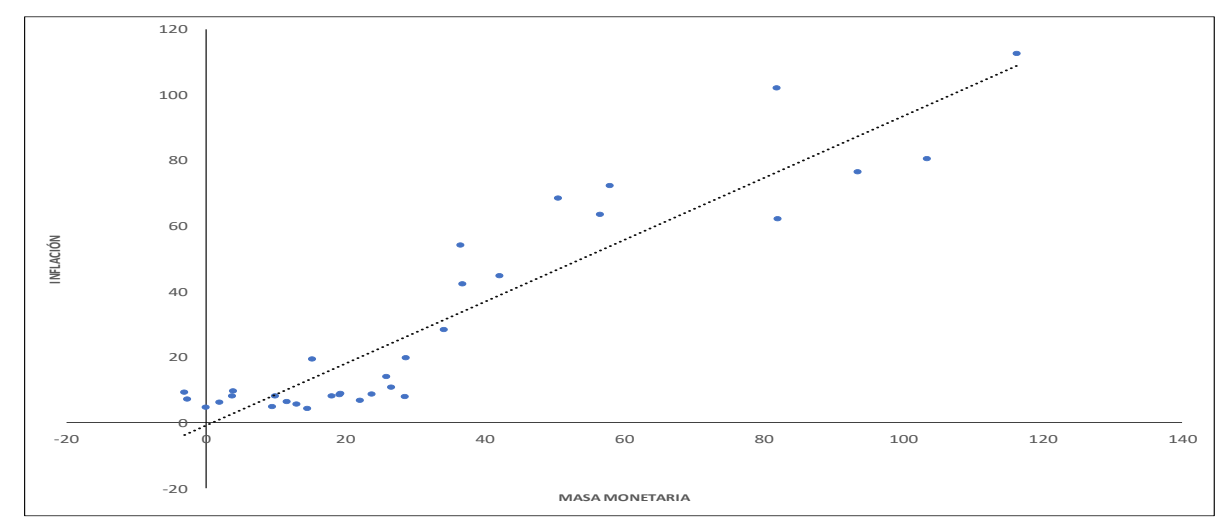

Fig. 2. Significancia del coeficiente de determinación

Fuente: elaboración propia

En la siguiente Figura 02 se observa la gráfica de dispersión, no existen datos muy dispersos por lo consiguiente el coeficiente de determinación $\left(R^{2}\right)$ tiene significancia, demostrando una vez más la relación directamente proporcional entre inflación y masa, entendiendo que un modelo adecuado para el tipo de variantes aplicadas

\section{Conclusiones}

El objetivo de este trabajo es explicar la incidencia de la inflación en la masa monetaria de Uruguay, durante un período de 1985-2017, para el presente trabajo se utilizó un modelo de regresión lineal simple con Mínimos Cuadrados Ordinarios, es importante mencionar que se utilizaron dos variables influyentes como la Inflación y la Masa monetaria. 


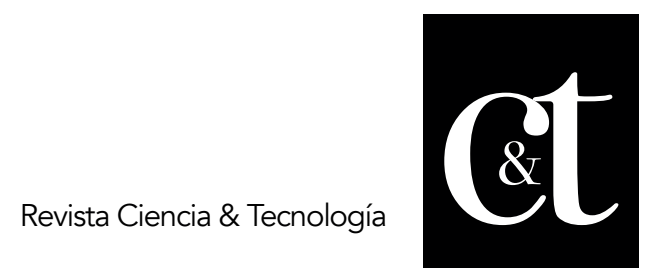

No. 30, 30 de abril de 2021

ISSN impreso: 1390 - 6321

ISSN online: 2661 - 6734

Los resultados obtenidos fueron significativos, es decir que las variantes están relacionadas, el coeficiente de determinación fue clave para el modelo al tener un valor dentro de lo permitido como es 0.8625 , a medida que aumenta la inflación la masa monetaria aumentara o viceversa, los dos supuestos JarqueBera y Breusch-Pagan corroboraron aún más la propuesta determinando existir homocedasticidad y normalidad en los residuos.

En base a lo anterior, el comportamiento de la inflación ha sido relevante por los valores agresivos desde 1985 generados por la incertidumbre en la inversión y consumo Uruguayo, dificultades que llevaron a predecir las ganancias e ingresos futuros; desde 1990 la visión monetarista tuvo un movimiento en función de la demanda real de dinero, pues el fenómeno inflacionario provocó choques y aumentos vertiginosos por la inyección de divisas.

La teoría cuantitativa del dinero ratifica la relación directa de las dos variables al producir un aumento o disminución de la oferta monetaria dado por la velocidad del dinero y la producción acontecida en el presente de estudio.

Finalmente, los problemas económicos presentados al principio se convirtieron en moderados crecimientos económicos desde 1991 hasta 2016 y 2017, la solución del modelo en el equilibrio de largo plazo impactó seriamente al sistema económico, sin embargo, la competitividad de la economía permitió mejorar las expectativas por la estabilidad en los sistemas de precios, especialmente en los salarios nominales y exportaciones con términos de intercambio resultados favorables de las políticas económicas adoptadas.

Así mismo, los importantes avances en materia de estabilidad macroeconómica, generaron ganancias en el crecimiento a largo plazo a través de las disminuciones de la tasa de inflación, a pesar que la masa monetaria bajó en porcentuales menores significaron un alcance favorable para la economía y el frondoso crecimiento económico de Uruguay. Estos acontecimientos permiten visualizar la construcción de una economía Latinoamericana ante escenarios fortuitos cuando la gobernanza pública considera como prioridad salvaguardar el desarrollo económico de los agentes económicos.

\section{Referencias}

Astorga, J. (2014). Aplicación de modelos de regresión lineal para determinar las armónicas de tensión y corriente. Ingeniería Energética, 234-244.

Banco Interamericano de Desarrollo (BID). (2015). Crecimiento Económico y Brechas de Desarrolllo en Uruguay. Obtenido de Crecimiento Económico y Brechas de Desarrolllo en Uruguay: https://publications.iadb.org/publications/spanish/document/Crecimientoecon\%C3\%B3mico-y-brechas-de-desarrollo-en-Uruguay.pdf

Barquero, J., y Loaiza, K. (2017). Inflación y deuda pública. Monetaria, 44-100.

León, Llaguno, Recalde. Uruguay: Análisis econométrico de la masa monetaria e inflación, $1985-2017$ 


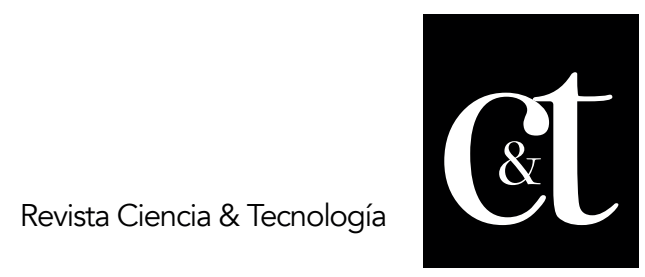

No. 30, 30 de abril de 2021

ISSN impreso: 1390 - 6321

ISSN online: 2661 - 6734

Brum, C., Román, C., y Willebald, H. (2013). Inflación en Uruguay en 140 años de historia (1870-2010). Un enfoque monetario. Obtenido de Banco Central del

Uruguay: https://www.bcu.gub.uy/Comunicaciones/Jornadas\%20de\%20Economa/t_br um_conrado_2013_1632.pdf

Calderón, K. (2015). Inflación y su impacto en la lectura y análisis de estados financieros en la toma de decisiones. Repositorio Digital Universidad Nacional de Córdoba, 1-89.

Campoverde, J. (2019). Determinantes de la oferta monetaria en una economía dolarizada: Caso ecuatoriano periodo 2000-2017. Repositorio Institucional Universidad del Azuay, 1-137.

Cauas, D. (2015). Definición de las variables enfoque y tipo de investigación. Academia Amazonas, 1-11.

Chraki, F. (2018). Valor y Dinero en Marx. Revista de Economía Institucional, 131.

Fondo Monetario Internacional (FMI). (2017). Uruguay: Declaración del personal del FMI al término de la misión sobre la Consulta del Artículo IV de 2017. Obtenido de https://www.imf.org/es/News/Articles/2017/12/07/ms120717uruguay-staff-concluding-statement-of-the-2017-article-iv-mission

Fischer, Stanley, y Easterly William (1990). The Economics of the Government Budget Constraint, World Bank Research Observer, vol. 5, núm. 2, julio, $127-142$

Gómez, L. (2017). Estudio de la relación funcional entre el nivel de inflación y la demanda de dinero real. Centro Universitario UAEM Tenancingo, 85.

Gujarati, D., y Porter, D. (2012). Econometría. Ciudad de México: McGRAWHILL/INTERAMERICANA EDITORES, S.A. DE C.V.

Lage, C. (2014). Planificación monetaria: un acercamiento desde la teoría monetaria en Marx. Redalyc, 1-15.

Laguna, C. (2014). Correlación y regresión lineal. Instituto Aragonés de Ciencia de la Salud, 1-18.

León, L. (2018). Análisis econométrico de la inflación y su incidencia en el desempleo ecuatoriano, período 1990-2016. Espacios, 39(32), 1-9.

Luceño, A., \& Gonzáles, F. (2015). Método estadístico para medir ,describir y controlar la variabilidad. Santander: Editorial Universidad de Cantabria.

Mankiw, G. (2014). Macroeconomía. España: Antoni Bosch editor.

Mañan, O. (2010). Uruguay: crisis, inflexión Uruguay: crisis, inflexión. Revista Política y cultura(34), 213-226.

Mballa, V., y Sauceda, A. (2018). Análisis del hambre en el estado de Zacatecas bajo el modelo de Mínimos Cuadrados Ordinarios. Economía Sociedad y Territorio, 487-523.

Mcleay, M., Radia, A., y Thomas, R. (2015). El dinero en la economía moderna: una introducción. Revista de Economía Institucional, 1-21.

Mundial, B. (2019). Banco Mundial. Obtenido https://datos.bancomundial.org/indicador/FP.CPI.TOTL.ZG?locations=CL

Parejo, F. (2015). El dinero en la historia del pensamiento económico. Universidad de Extremadura, 15.

Ravier, A. (2017). Virtudes y límites de la teoría cuantitativa del dinero. Papers .SSRN, 1-15. 


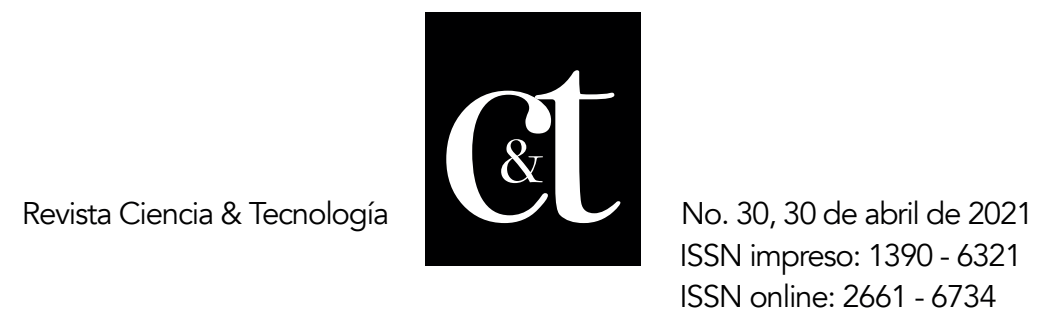

Romo, S., y Campi, A. (2018). Influencias de las variables representativas del sectro externo en la liquides Ecuatoriana para el periodo 2000-2016. Repositorio Digital de la Universidad Central del Ecuador, 1-13.

Stock, J., y Watson, M. (2012). Introduccion a la econometria. Colombia: Pearson Eduacación SA.

Universidad de la República. (2010). Políticaa Monetaria y Oferta de Dinero en Uruguay (1931-1959): Nuevos contextos y nuevos instrumentos. Instituto de Economía, Facultad de Ciencias Económicas y de Administración. Obtenido de http://codexvirtual.com/hbancaria/wpcontent/plugins/documentos/files/Politica_monetaria_y_oferta_de_dinero_en Uruguay.pdf

Vallejo, L. (2016). La inflación en Colombia. Revista de Apuntes del CENES, 910.

Wooldridge, j. (2008). Introducción a la econometría: Un enfoque moderno. Madrid: Thomson Paraninfo S.A.

León, Llaguno, Recalde. Uruguay: Análisis econométrico de la masa monetaria e inflación, 1985 - 2017 